\title{
Dense Relations Are Determined by Their Endomorphism Monoids
}

\author{
João Araújo and Janusz Konieczny
}

\begin{abstract}
We introduce the class of dense relations on a set $X$ and prove that for any finitary or infinitary dense relation $\rho$ on $X$, the relational system $(X, \rho)$ is determined up to semi-isomorphism by the monoid $\operatorname{End}(X, \rho)$ of endomorphisms of $(X, \rho)$. In the case of binary relations, a semi-isomorphism is an isomorphism or an anti-isomorphism.
\end{abstract}

2000 Mathematics Subject Classification: 20M20, $20 \mathrm{M} 15$.

\section{Introduction}

For a mathematical structure $M$, let $\operatorname{End}(M)$ denote the endomorphism monoid of $M$. A general problem, which attracted a considerable attention, can be stated as follows: Let $M_{1}, M_{2}$ be two mathematical structures. Given that $\operatorname{End}\left(M_{1}\right) \cong \operatorname{End}\left(M_{2}\right)$, what can we say about the relation between the structures $M_{1}$ and $M_{2}$ themselves? For example, Schein [8] proved that if $M_{1}$ and $M_{2}$ are two partially ordered sets, semilattices, distributive lattices, or Boolean algebras, then $\operatorname{End}\left(M_{1}\right) \cong \operatorname{End}\left(M_{2}\right)$ if and only if $M_{1}$ and $M_{2}$ are isomorphic or anti-isomorphic. For other results of this kind, see [2], [4], and [5].

The aim of this note is to prove a similar result for the class of dense relations. These relations include partial orders, binary relations that are reflexive and symmetric, and generalized equivalence relations.

Let $I$ be an arbitrary non-empty index set. An $I$-tuple of elements of a set $X$ is a mapping $f: I \rightarrow X$. If $I$ is finite with $|I|=n$, we shall assume that $I=\{1,2, \ldots, n\}$, denote $f: I \rightarrow X$ by $(1 f, 2 f, \ldots, n f)$, and refer to $f$ as an $n$-tuple. An $I$-relation $\rho$ on $X$ is any set of $I$-tuples of elements of $X$. If $|I|=n$, an $I$-relation $\rho$ is an $n$-ary relation on $X$, that is, a set of $n$-tuples of elements of $X$. By a relation on $X$, we shall mean an $I$-relation on $X$ for some index set $I$.

Let $\rho$ be an $I$-relation on $X$. An endomorphism of a relational system $(X, \rho)$ is a mapping $a: X \rightarrow X$ that preserves $\rho$, that is, fa $\in \rho$ for every $f \in \rho$, where $f a: I \rightarrow X$ is the composition of $f: I \rightarrow X$ and $a: X \rightarrow X$. (We compose from left to right, that is, $i(f a)=(i f) a$ for $i \in I$.) If $\rho$ is an $n$-ary relation, we can use the $n$-tuple notation. With that notation, we have that $a: X \rightarrow X$ is an endomorphism of $(X, \rho)$ if and only if $\left(x_{1} a, \ldots, x_{n} a\right) \in \rho$ for every $\left(x_{1}, \ldots, x_{n}\right) \in \rho$. We denote by End $(X, \rho)$ the monoid of endomorphisms of $(X, \rho)$.

Turning to the definition of a dense relation, we denote by $\rho^{*}$ the set of all mappings $f: I \rightarrow X$ such that $\sigma f \notin \rho$ for every $\sigma \in S(I)$, where $S(I)$ is the symmetric group of $I$. 
That is,

$$
\rho^{*}=\{f: I \rightarrow X:(\forall \sigma \in S(I)) \sigma f \notin \rho\} .
$$

A reflexive $I$-relation $\rho$ on $X$ is said to be dense if it satisfies the following two properties:

$\left(D_{1}\right)$ For every injective $f_{1} \in \rho \cup \rho^{*}$ and every $f \in \rho$, there is $a \in \operatorname{End}(X, \rho)$ such that $f_{1} a=f$.

$\left(D_{2}\right)$ There is an injective $f_{1}$ in $\rho$.

Examples of dense relations (for details see [1]) are partial orders, binary relations that are reflexive and symmetric, generalized equivalence relations (see [6]), relations defined by families of sets intersecting in at most one element (see [7]).

Let $\rho_{1}, \rho_{2}$ be $I$-relations on $X_{1}, X_{2}$, respectively. We say that a bijection $g: X_{1} \rightarrow X_{2}$ is a semi-isomorphism of $\left(X_{1}, \rho_{1}\right)$ to $\left(X_{2}, \rho_{2}\right)$ if there is a permutation $\sigma \in S(I)$ such that for all $f: I \rightarrow X_{1}$,

$$
f \in \rho_{1} \Leftrightarrow \sigma f g \in \rho_{2} .
$$

We say that relational systems $\left(X_{1}, \rho_{1}\right)$ and $\left(X_{2}, \rho_{2}\right)$ are semi-isomorphic if there is a semi-isomorphism from $\left(X_{1}, \rho_{1}\right)$ to $\left(X_{2}, \rho_{2}\right)$.

Note that if $I=\{1,2\}$ then the only elements of $S(I)$ are $i d_{I}$ (the identity permutation of $I$ ) and the transposition (1 2). It follows that if $\rho_{1}$ and $\rho_{2}$ are binary relations then any semi-isomorphism $g: X_{1} \rightarrow X_{2}$ is either an isomorphism $\left((x, y) \in \rho_{1} \Leftrightarrow(x g, y g) \in\right.$ $\left.\rho_{2}\right)$ or an anti-isomorphism $\left((x, y) \in \rho_{1} \Leftrightarrow(y g, x g) \in \rho_{2}\right)$.

In the next section, we prove that if $\rho$ is a dense $I$-relation on $X$ then $\operatorname{End}(X, \rho)$ determines $\rho$ up to a semi-isomorphism.

\section{Main Theorem and Its Application to Binary Relations}

The following lemma belongs to the folklore (see [2], for example) and we include a proof just for the sake of completeness.

Lemma 2.1 Let $\rho_{1}, \rho_{2}$ be reflexive I-relations on $X_{1}, X_{2}$, respectively, and suppose that $\phi: \operatorname{End}\left(X_{1}, \rho_{1}\right) \rightarrow \operatorname{End}\left(X_{2}, \rho_{2}\right)$ is an isomorphism. Then there exists a bijection $g:$ $X_{1} \rightarrow X_{2}$ such that $a \phi=g^{-1}$ ag for every $a \in \operatorname{End}\left(X_{1}, \rho_{1}\right)$.

Proof: For a set $X$ and $x \in X$, we denote by $X_{x}$ the constant mapping from $X$ to $X$ defined by: $y X_{x}=x$ for every $y \in X$. Let $S$ be a semigroup of mappings from $X$ to $X$ with $X_{x} \in S$ for some $x \in X$. It is easy to see that $\left\{X_{x}\right\}$ is a minimal left ideal of $S$. In fact, all minimal left ideals of $S$ are of the form $\left\{X_{y}\right\}$ where $y \in X$. For suppose $J$ is a minimal left ideal of $S$ and let $a \in J$. Then $X_{x} a \in J$ and $X_{x} a=X_{x a}$. Since $\left\{X_{x a}\right\} \subseteq J$ and $J$ is a minimal left ideal, it follows that $\left\{X_{x a}\right\}=J$. Denote by $\mathcal{M}(S)$ the set of minimal left ideals of $S$. We proved that $\mathcal{M}(S)=\left\{\left\{X_{x}\right\}: X_{x} \in S\right\}$.

Since $\operatorname{End}\left(X_{1}, \rho_{1}\right)$ and $\operatorname{End}\left(X_{2}, \rho_{2}\right)$ contain all constant mappings on $X_{1}$ and $X_{2}$, respectively, we have $\mathcal{M}\left(\operatorname{End}\left(X_{1}, \rho_{1}\right)\right)=\left\{\left\{\left(X_{1}\right)_{x}\right\}: x \in X_{1}\right\}$ and $\mathcal{M}\left(\operatorname{End}\left(X_{2}, \rho_{2}\right)\right)=$ $\left\{\left\{\left(X_{2}\right)_{y}\right\}: y \in X_{2}\right\}$. Since an isomorphism must map minimal left ideals to minimal left ideals, it follows that $\left\{\left(X_{1}\right)_{x} \phi: x \in X_{1}\right\}=\left\{\left(X_{2}\right)_{y}: y \in X_{2}\right\}$, so that $\left|X_{1}\right|=\left|X_{2}\right|$. Define $g: X_{1} \rightarrow X_{2}$ by: $x g=y$ if $\left(X_{1}\right)_{x} \phi=\left(X_{2}\right)_{y}$. Then $g$ is a bijection and $\left(X_{1}\right)_{x} \phi=\left(X_{2}\right)_{x g}$ for every $x \in X_{1}$. 
Let $x \in X_{1}$ and $a \in \operatorname{End}\left(X_{1}, \rho_{1}\right)$. We claim that $(x g)(a \phi)=(x a) g$. Indeed, $(x g)\left(X_{2}\right)_{x g}(a \phi)=(x g)\left(\left(X_{1}\right)_{x} \phi\right)(a \phi)=(x g)\left(\left(X_{1}\right)_{x} a\right) \phi=(x g)\left(\left(X_{1}\right)_{x a} \phi\right)=(x g)\left(X_{2}\right)_{(x a) g}$. Therefore $(x g)(a \phi)=(x g)\left(X_{2}\right)_{x g}(a \phi)=(x g)\left(X_{2}\right)_{(x a) g}=(x a) g$. It follows that $g(a \phi)=$ $a g$ and hence $a \phi=g^{-1} a g$.

Note that if $g$ is as in the statement of Lemma 2.1 then for all $a \in \operatorname{End}\left(X_{1}, \rho_{1}\right)$ and $b \in \operatorname{End}\left(X_{2}, \rho_{2}\right), g^{-1} a g \in \operatorname{End}\left(X_{2}, \rho_{2}\right)$ and $g b g^{-1} \in \operatorname{End}\left(X_{1}, \rho_{1}\right)$.

Lemma 2.2 Let $\rho_{1}, \rho_{2}$ be dense I-relations on $X_{1}, X_{2}$, respectively, and let $g: X_{1} \rightarrow X_{2}$ be a bijection such that $g^{-1} a g \in \operatorname{End}\left(X_{2}, \rho_{2}\right)$ for every $a \in \operatorname{End}\left(X_{1}, \rho_{1}\right)$. Then for every $f \in \rho_{1}$ there is a permutation $\sigma \in S(I)$ such that $\sigma f g \in \rho_{2}$.

Proof: Fix an injective $f_{2} \in \rho_{2}$ (which exists by $\left(D_{2}\right)$ ) and let $f_{1}=f_{2} g^{-1}$. Note that $f_{1}$ is injective and $f_{2}=f_{1} g$. Let $f$ be an arbitrary element of $\rho_{1}$.

Suppose $f_{1} \in \rho_{1}^{*}$. Then, by $\left(D_{1}\right)$, there is $a \in \operatorname{End}\left(X_{1}, \rho_{1}\right)$ such that $f_{1} a=f$. Thus, since $g^{-1} a g \in \operatorname{End}\left(X_{2}, \rho_{2}\right)$, we have

$$
f_{2} \in \rho_{2} \Rightarrow f_{2}\left(g^{-1} a g\right) \in \rho_{2} \Rightarrow f_{1} a g \in \rho_{2} \Rightarrow f g \in \rho_{2} \Rightarrow i d_{I} f g \in \rho .
$$

Suppose $f_{1} \notin \rho_{1}^{*}$. Then $\sigma f_{1} \in \rho_{1}$ for some $\sigma \in S(I)$. By $\left(D_{1}\right)$, there is $a \in$ End $\left(X_{1}, \rho_{1}\right)$ such that $\sigma f_{1} a=f$. Then $f_{1} a=\sigma^{-1} f$ and we have

$$
f_{2} \in \rho_{2} \Rightarrow f_{2}\left(g^{-1} a g\right) \in \rho_{2} \Rightarrow f_{1} a g \in \rho_{2} \Rightarrow \sigma^{-1} f g \in \rho_{2} .
$$

The lemma follows.

Lemma 2.3 With the hypothesis of Lemma 2.2, let $f_{1} \in \rho_{1}$ be injective and let $\sigma \in S(I)$. If $\sigma f_{1} g \in \rho_{2}$ then $\sigma f g \in \rho_{2}$ for every $f \in \rho_{1}$.

Proof: Suppose $\sigma f_{1} g \in \rho_{2}$ and let $f \in \rho_{1}$. By $\left(D_{1}\right)$, there is $a \in \operatorname{End}\left(X_{1}, \rho_{1}\right)$ such that $f_{1} a=f$. Since $g^{-1} a g \in \operatorname{End}\left(X_{2}, \rho_{2}\right)$, we have

$$
\sigma f_{1} g \in \rho_{2} \Rightarrow \sigma f_{1} g\left(g^{-1} a g\right) \in \rho_{2} \Rightarrow \sigma f_{1} a g \in \rho_{2} \Rightarrow \sigma f g \in \rho_{2},
$$

which concludes the proof.

We can now prove the main theorem of this note.

Theorem 2.4 Let $\rho_{1}, \rho_{2}$ be dense I-relations on $X_{1}, X_{2}$, respectively. Then the endomorphism monoids End $\left(X_{1}, \rho_{1}\right)$ and End $\left(X_{2}, \rho_{2}\right)$ are isomorphic if and only if the relational systems $\left(X_{1}, \rho_{1}\right)$ and $\left(X_{2}, \rho_{2}\right)$ are semi-isomorphic.

Proof: Suppose $\phi: \operatorname{End}\left(X_{1}, \rho_{1}\right) \rightarrow \operatorname{End}\left(X_{2}, \rho_{2}\right)$ is an isomorphism. By Lemma 2.1, there is a bijection $g: X_{1} \rightarrow X_{2}$ such that $a \phi=g^{-1} a g$ for every $a \in \operatorname{End}\left(X_{1}, \rho_{1}\right)$. We claim that $g$ is a semi-isomorphism from $\left(X_{1}, \rho_{1}\right)$ to $\left(X_{2}, \rho_{2}\right)$. Since $\rho_{1}$ and $\rho_{2}$ are dense, there are $f_{1} \in \rho_{1}$ and $f_{2} \in \rho_{2}$ such that $f_{1}$ and $f_{2}$ are injective. By Lemma 2.2, there is $\sigma \in S(I)$ such that $\sigma f_{1} g \in \rho_{2}$. By Lemma 2.3, $\sigma f g \in \rho_{2}$ for every $f \in \rho_{1}$. Now, note that $f_{2}^{\prime}=\sigma f_{1} g$ is an injective element of $\rho_{2}$ and $\sigma^{-1} f_{2}^{\prime} g^{-1}=f_{1} \in \rho_{1}$. Thus, by 
Lemma 2.3, $\sigma^{-1} f^{\prime} g^{-1} \in \rho_{1}$ for every $f^{\prime} \in \rho_{2}$. It follows that for every $f: I \rightarrow X_{1}$, $f \in \rho_{1} \Leftrightarrow \sigma f g \in \rho_{2}$, and so $g$ is a semi-isomorphism.

Conversely, suppose that $g: X_{1} \rightarrow X_{2}$ is a semi-isomorphism, and let $\sigma \in S(I)$ be such that for every $f: I \rightarrow X_{1}, f \in \rho_{1} \Leftrightarrow \sigma f g \in \rho_{2}$. Then for all $a \in \operatorname{End}\left(X_{1}, \rho_{1}\right)$ and $f^{\prime}: I \rightarrow X_{2}$,

$$
f^{\prime} \in \rho_{2} \Rightarrow \sigma^{-1} f^{\prime} g^{-1} \in \rho_{1} \Rightarrow \sigma^{-1} f^{\prime} g^{-1} a \in \rho_{1} \Rightarrow \sigma \sigma^{-1} f^{\prime} g^{-1} a g \in \rho_{2} \Rightarrow f^{\prime} g^{-1} a g \in \rho_{2} .
$$

It follows that $\phi: \operatorname{End}\left(X_{1}, \rho_{1}\right) \rightarrow \operatorname{End}\left(X_{2}, \rho_{2}\right)$ defined by: $a \phi=g^{-1} a g$ is an isomorphism.

Applying Theorem 2.4 to binary relations, we obtain the following corollary.

Corollary 2.5 Let $\rho_{1}, \rho_{2}$ be binary dense relations on $X_{1}, X_{2}$, respectively. Then the endomorphism monoids End $\left(X_{1}, \rho_{1}\right)$ and End $\left(X_{2}, \rho_{2}\right)$ are isomorphic if and only if the relational systems $\left(X_{1}, \rho_{1}\right)$ and $\left(X_{2}, \rho_{2}\right)$ are isomorphic or anti-isomorphic.

Problem Classify the $n$-ary dense relations, for $n \geq 2$.

\section{References}

[1] J. Araújo and J. Konieczny, A method for finding automorphism groups of endomorphism monoids of relational and algebraic systems, to appear.

[2] L.M. Gluskinn, Semi-groups of isotone transformations, Uspehi Mat. Nauk 16 (1961), 157-162. (Russian)

[3] J.M. Howie, "Fundamentals of Semigroup Theory," Oxford University Press, New York, 1995.

[4] G. Mashevitzky and B.M. Schein, Automorphisms of the endomorphism semigroup of a free monoid or a free semigroup, Proc. Amer. Math. Soc. 131 (2003) 1655-1660.

[5] V.A. Molchanov, Semigroups of mappings on graphs, Semigroup Forum 27 (1983), 155-199.

[6] H.E. Pickett, A note on generalized equivalence relations, Amer. Math. Monthly 73 (1966), 860-861.

[7] H.J. Ryser, Subsets of a finite set that intersect each other in at most one element, J. Combinatorial Theory Ser. A 17 (1974), 59-77.

[8] B.M. Schein, Ordered sets, semilattices, distributive lattices and Boolean algebras with homomorphic endomorphism semigroups, Fund. Math. 68 (1970), 31-50.

J. Araújo

Universidade Aberta and CAUL

R. Escola Politécnica, 147

1269-001 Lisboa, Portugal

mjoao@lmc.fc.ul.pt
J. Konieczny

University of Mary Washington

Department of Mathematics

Fredericksburg, VA 22401, USA

jkoniecz@umw.edu 